Development and online operation of minimum bias triggers in ATLAS

This article has been downloaded from IOPscience. Please scroll down to see the full text article. 2010 JINST 5 C12051

(http://iopscience.iop.org/1748-0221/5/12/C12051)

View the table of contents for this issue, or go to the journal homepage for more

Download details:

IP Address: 137.138.124.142

The article was downloaded on 11/07/2011 at 13:54

Please note that terms and conditions apply. 
Topical Workshop on Electronics for Particle Physics 2010, 20-24 SEPTEMBER 2010,

\title{
Development and online operation of minimum bias triggers in ATLAS
}

\author{
T. Martin ${ }^{1}$ \\ School of Physics and Astronomy, University of Birmingham, \\ B15 2TT, United Kingdom \\ E-mail: tim.martin@cern.ch
}

ABSTRACT: Since the restart of the LHC in November 2009, ATLAS has recorded $p p$ collisions to investigate new phenomena at the high energy frontier. One early measurement is of charged particle multiplicities in inelastic interactions, which was performed on data sets of about 360,000 and $10,000,000$ minimum bias event at $\sqrt{s}=900 \mathrm{GeV}$ and $7 \mathrm{TeV}$ respectively. This measurements will help to constrain various phenomenological models describing inelastic interactions. Understanding trigger efficiencies for different event types is crucial to minimize any possible bias in the event selection. ATLAS has a three level trigger system and utilises two main minimum bias triggers. A primary scintillator based trigger situated in the forward regions of $2.1<|\eta|<3.8$ was proven to select $p p$ collisions with high efficiency. A complementary control trigger runs on unbiased filled proton bunches and utilizes space points reconstructed in the silicon tracking volume to trigger on inelastic interactions. Both triggers were essential for the analysis of charged particle multiplicities. Their performance and trigger efficiency measurements as well as studies on possible bias sources are presented. The triggers are shown to form an effective and inclusive triggering solution for early LHC physics.

KEYWORDS: Trigger concepts and systems (hardware and software); Trigger detectors

\footnotetext{
${ }^{1}$ On behalf of the ATLAS collaboration.
} 


\section{Contents}

1 Introduction 1

2 The ATLAS inner detector and trigger system 1

3 Minimum bias trigger scintillators $\quad 2$

4 Efficiency of the MBTS 3

5 Conclusion 4

\section{Introduction}

These proceedings document the infrastructure and performance of the Minimum Bias Trigger systems installed within the ATLAS detector at the LHC. The ATLAS detector [1] is a general purpose particle detector designed to study high energy $p p$ and heavy ion collisions. The first physics measurements made with ATLAS were of the properties of charged particles in $p p$ collisions at $\sqrt{s}=900 \mathrm{GeV}$ and $7 \mathrm{TeV}$ [2]. Inelastic diffractive and non-diffractive interactions form a major component of the total $p p$ cross section. Theoretically, inelastic diffraction is best described by phenomenological models while inelastic non-diffractive interactions are best described by lowest order QCD perturbative two-to-two scatters with phenomenological renormalisation of divergences. Consequently when extrapolated to LHC energies, minimum bias predictions carry large theoretical uncertainties and are model dependent. By recording events with minimum bias, maximally inclusive results were published with minimal model dependencies. Inelastic interactions were selected during early running, the performance of the trigger is determined using a complimentary control trigger. This is based upon activity recorded in the inner silicon tracking volumes.

\section{The ATLAS inner detector and trigger system}

ATLAS has a wide range of tracking, calorimetric and spectroscopic subdetectors. Of particular importance to the Minimum Bias Trigger are the Minimum Bias Trigger Scintillators (MBTS) and the silicon based inner detector trackers. The MBTS is discussed in detail in the next chapter.

The Inner Detector in ATLAS has a coverage of $|\eta|<2.5$. Closest to the interaction region lies the Pixel Detector, this consists of 3 barrel and 3 end cap layers of $50 \times 400 \mu \mathrm{m}$ pitch pixels with a resolution of $10 \mu \mathrm{m}$ in $r-\phi$ and $115 \mu \mathrm{m}$ in $z$. The Semiconductor Tracker (SCT) is positioned outside the Pixel detector, it consists of 4 barrel layers of $80 \mu \mathrm{m}$ pitch strips and 9 end cap layers. Each layer is formed of two strip detectors, sandwiched back-to-back with a stereo angle of 40 mrad providing $17 \mu \mathrm{m}$ resolution in $r-\phi$ and $580 \mu \mathrm{m}$ in $z$.

ATLAS has a 3-level trigger system. At Level 1 (L1), readout from the detector is held in a pipeline while fast trigger signals are sent to the Central Trigger Processor (CTP) where they are 
combined to form a trigger decision. The CTP forms trigger items from MBTS counters above threshold, trigger items include L1_MBTS_1, L1_MBTS_2, L1_MBTS_1_1 and L1_MBTS_4_4 which correspond to 'at least one MBTS counter', 'at least two MBTS counters on one side', 'at least one MBTS counter on each side' and 'at least four MBTS counters on each side' respectively. Trigger bias is increased by both requiring multiple counters and by requiring counters on each side of the MBTS.

The High Level Trigger (HLT) consists of the Level 2 (L2) and the Event Filter (EF). The Inner Detector Minimum Bias Trigger (ID Mb) runs on the HLT, ID Mb algorithms at L2 are seeded from random triggers. The random trigger logic is internal to the CTP, it selects events at random when both beams are present in the detector. The L2 algorithms can only process data from all of the silicon tracking volume at a limited rate, prescale factors are therefore applied at L1 to limit the rate of the random trigger based on running conditions. The algorithms read in all hits from the Pixel and SCT sub detectors. Clustered pixel hits form pixel space points, while space points in the SCT are formed by two back-to-back stereo SCT hits. Events are accepted at L2 based on the multiplicity of Pixel and SCT space points; this requirement vetoes empty events while maintaining a high efficiency for collisions. The configuration was adjusted during $7 \mathrm{TeV}$ running so as to remain fully efficient. In July 2010 two SCT clusters and two Pixel clusters were required. At the Event Filter, tracking algorithms are run to reconstruct tracks with the ability to cut on their longitudinal impact parameter with respect to the nominal interaction point. This EF stage was designed to veto beam background such as beam-gas interactions, these collisions occur uniformly along the beam line due to a constant vacuum pressure. This trigger is useful in the event of high rates of beam-gas, however beam backgrounds were investigated and shown to be low so ID Mb algorithms at the EF are run in pass-through mode. By running the algorithms in pass-through, the results of the algorithms are recorded for subsequent monitoring and analysis but are not used to actively reject events at the EF.

The efficiency of ID Mb was determined with respect to a control sample triggered by L1_MBTS_2 with offline track requirements detailed in [4]. It was observed that ID Mb is highly efficient down to low track multiplicities. This uniformly high efficiency means that the trigger is effectively unbiased to the number of offline selected tracks making it an excellent control trigger. The efficiency is shown in figure 2 [3].

\section{Minimum bias trigger scintillators}

The MBTS consists of planes of $2 \mathrm{~cm}$ thick polystyrene scintillator located at $\pm 3560 \mathrm{~mm}$ from the nominal interaction point in $z$ which are segmented around the beam pipe, perpendicular to the beam axis. Each side of the MBTS is divided into two segments laterally, covering pseudo rapidity $2.82<|\eta|<3.84$ and $2.09<|\eta|<2.82$ respectively and into 8 equal azimuthal segments resulting in 16 individual counters per side. Each counter is read out via wavelength shifting fibres embedded within the plastic connecting to photomultiplier tubes of adjustable high voltage. The photomultipliers are connected to a patch panel where the differential signals are converted to single ended signals by Low Voltage Differential Signal to Transistor-transistor Logic converter boards.

In order to provide a fast trigger signal, standard NIM electronics are used to amplify the signals with a minimum gain of $x 2$. The analogue signals are fed into 32 channels of Leading Edge 


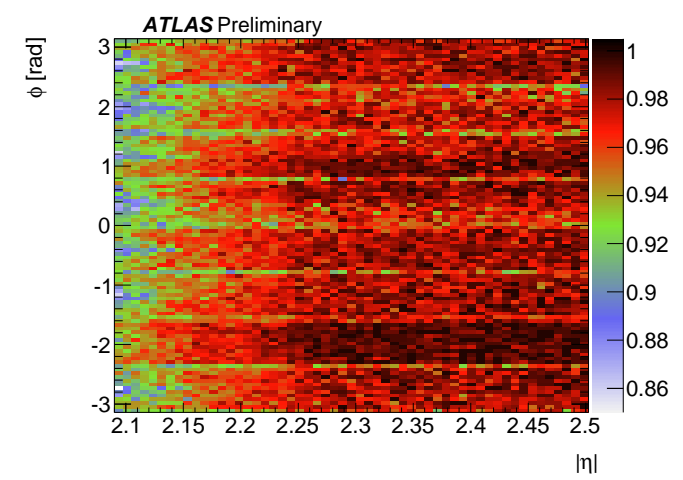

Figure 1. Efficiency of one side of the MBTS covering the inner detector overlap region in data. Efficiency is defined in the text.

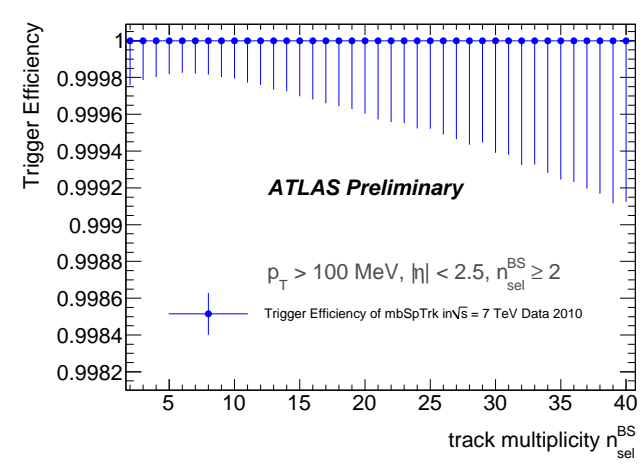

Figure 2. Efficiency of the ID Mb trigger as a function of the number of offline selected tracks. The trigger is shown to be unbiased to the number of offline reconstructed tracks.

(LE) discriminators, the output logic signal is used as an input to the CTP. The discriminator thresholds are set to trigger on the signal from a minimum ionizing particle traversing a MBTS counter perpendicular to its surface. Being LE discriminators, some time walk is possible depending on the magnitude of the signal. A time walk of up $50 \mathrm{~ns}$ was measured using cosmic ray particles, therefore pulse stretching is used both in the LE discriminators and the CTP. To insure the CTP latches, the LE discriminators stretch the pulse over two bunch crossings (50 ns), the CTP then additionally stretches the pulses by \pm 4 bunch crossings before counter multiplicities are calculated. More recently, the spacing between filled bunches has reduced due to the introduction of bunch trains and the CTP stretching for the MBTS was reduced to \pm 2 bunch crossings.

For commissioning in 2009, the MBTS multiplicity bits were combined with the electrostatic Beam Pickup Monitors, hence ensuring that only the bunch crossing when two potentially colliding beams were in ATLAS is triggered for physics. In 2010 ATLAS commissioned bunch groups, the bunch group mechanism enables the selection of bunches that are colliding within ATLAS. It is set to match the LHC fill pattern of each run, MBTS bits are now combined with the appropriate bunch groups to ensure the MBTS is in time with colliding proton bunches.

The efficiency of the MBTS as a particle detector was measured by extrapolating the trajectory of tracks from the Inner Detector to the MBTS. The efficiency is presented as a function of the probe track $\eta$ and $\phi$ in figure 1 with the requirement that only one extrapolated track passes through a counter. When this criteria was met, the efficiency was defined as the fraction of probe tracks with a charge deposit of $15 \mathrm{pC}$ or more in the corresponding counter. The low efficiency at small $\eta$ is dominated by track extrapolation error.

\section{Efficiency of the MBTS}

The first physics publication [2] with collision data at a centre-of-mass energy of $900 \mathrm{GeV}$ by the ATLAS collaboration was on charged particle multiplicities in Minimum Bias events. Further results were published at an additional centre-of-mass energy of $7 \mathrm{TeV}$ and for a larger kinematic 


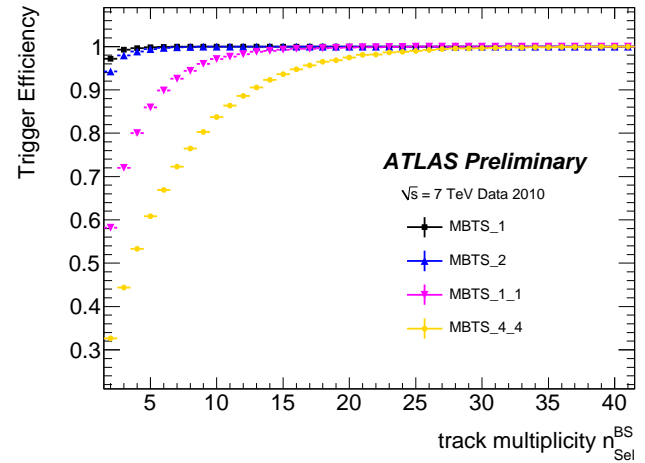

Figure 3. Comparison of the efficiency of various L1_MBTS triggers, determined from ID Mb. Only statistical errors are shown.

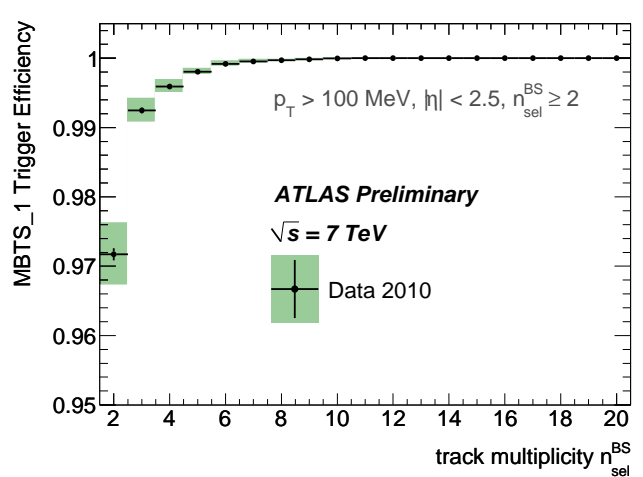

Figure 4. Efficiency of the L1_MBTS_1 trigger at $\sqrt{s}=7 \mathrm{TeV}$ as a function of the number of offline selected tracks. The shaded region denotes total uncertainty.

region of charged particles with $p_{\mathrm{T}}>100 \mathrm{MeV}$ and $|\eta|<2.5$ [4]. This analysis was performed using the L1_MBTS_1 trigger and therefore the results needed to be corrected for inefficiency in this trigger. This was achieved in a data-driven manner using ID Mb as the control trigger.

The efficiency of MBTS trigger items was calculated with respect to the control trigger, it was defined as

$$
\varepsilon(\text { L1_MBTS_X })=\frac{\text { L1_MBTS_X \& ID Mb \& Offline }}{\text { ID Mb \& Offline }} .
$$

Here L1_MBTS_X refers to either L1_MBTS_1, L1_MBTS_2, L1_MBTS_1_1 or L1_MBTS_4_4 and Offline refers to the offline track requirements for physics selection including track quality and impact parameter requirements. L1_MBTS_1 is the most inclusive but also the most susceptible to beam backgrounds, the two sided MBTS triggers are less prone to beam background but introduce more event selection bias. The trigger allows for flexible configuration of these items for quick adoption to changing beam background conditions. The efficiency is shown in figure 3 for these trigger items with statistical error only. In figure 4 the shaded area shows the statistical and systematic error on the efficiency of L1_MBTS_1. These are added in quadrature and the square root taken. Two sources of systematic error were considered. Systematic effects due to variation of impact parameter cuts from those in the offline selection were determined from data. This provides a conservative estimate of the effects of beam background and secondary tracks on trigger efficiency. Secondly the correlation between the selection (L1_MBTS_1) and the control trigger (ID Mb) was investigated. This was determined from Monte Carlo to be negligible.

\section{Conclusion}

The ATLAS Minimum Bias Trigger Scintillators have provided an effective and inclusive triggering solution for early LHC physics during a period of detector commissioning. The complimentary ID Mb trigger was used to quantify the efficiency of the MBTS and monitor beam background conditions within the detector. This has resulted in accurate new measurements of inelastic $p p$ interactions with minimal model dependence. 


\section{References}

[1] ATLAS collaboration, G. Aad et al.,Expected Performance of the ATLAS Experiment - Detector, Trigger and Physics [arXiv:0901.0512].

[2] ATLAS collaboration, Charged-particle multiplicities in pp interactions at $\sqrt{s}=900 \mathrm{GeV}$ measured with the ATLAS detector at the LHC, Phys Lett. B 688 (2010) 21 [arXiv : 1003. 3124].

[3] ATLAS collaboration, Performance of the Minimum Bias Trigger in p-p Collisions at $\sqrt{s}=7 \mathrm{TeV}$, ATLAS report ATLAS-CONF-2010-068.

[4] ATLAS collaboration, Charged particle multiplicities in pp interactions for track $p_{T}>100 \mathrm{MeV}$ at $\sqrt{s}=0.9$ and $7 \mathrm{TeV}$ measured with the ATLAS detector at the LHC, ATLAS report ATLAS-CONF-2010-046. 